\title{
sciendo
}

Transport and Telecommunication, 2021, volume 22, no. 3, 343-352

Transport and Telecommunication Institute, Lomonosova 1, Riga, LV-1019, Latvia

DOI 10.2478/ttj-2021-0027

\section{GENETIC ALGORITHM FOR THE PICK-UP AND DELIVERY PROBLEM WITH TIME WINDOW BY MULTI-COMPARTMENT VEHICLES}

\author{
Berte Ousmane ${ }^{1}$, Diaby Moustapha ${ }^{2}$, Coulibaly Adama ${ }^{3}$ \\ ${ }^{1}$ Université Felix Houphouët Boigny, Abidjan, côte d'ivoire \\ berteousmane2012@hotmail.fr \\ ${ }^{2}$ Ecole supérieure africaine des TIC, Abidjan, Cote d'Ivoire \\ Moustapha.diaby@esatic.edu.ci \\ ${ }^{3}$ Université Felix Houphouët Boigny, Abidjan, Cote d'Ivoire \\ couliba@yahoo.fr
}

\begin{abstract}
In this article we focus on multi-compartment vehicles that have to pick-up goods from suppliers and deliver them to various customers. Usually these goods cannot be transported by single-compartment vehicles due to the fact that some products are harmful to others: incompatibility between products. It is therefore a question of satisfying a set of customers while respecting the constraints linked to the capacity of each vehicle compartment, each type of product and ensuring that each supplier is visited before his customer. Our work consists firstly of mathematically modelling the problem described and secondly of solving it using methods due to its complexity. In this case we use the genetic algorithm to solve the pick-up and delivery problem of goods with a time window provided by multi-compartment vehicles. Our model allows to find a minimum distance and a minimum cost in the tour carried out by a reasonable number of vehicles.
\end{abstract}

Keywords: Genetic Algorithm, Multi-Compartment Vehicles, PDPTW

\section{Introduction}

In the management of a company, the cost of transporting goods between suppliers and customers is a factor of paramount importance in the supply chain. The advent of new technologies has favoured online purchasing. In this process, companies are responsible for collecting goods from suppliers and delivering them to their customers. During this process, the company seeks to minimise these expenses and to be efficient by respecting the time windows defined by its customers. In fact, it generally operates in a very competitive environment.

This transport service is confronted with various problems, in particular:

- environmental problems (emission of $\mathrm{CO}_{2}$ by several vehicles),

- vehicle management problems (numbers put into service for greater profitability),

- problems related to vehicle capacity and problems related to respecting the time interval given by each client in order to produce a quality service.

It should also be noted that delivery agents encounter several difficulties when unloading with single-compartment vehicles. During these unloading operations, they lose enough time unloading the goods at height, sorting the different products of this client. Moreover, very often some products are harmful to others, damage other products, exposed to food safety or hygiene problems.

In this article, in order to avoid all these problems mentioned above, we will address the problem of delivery with the use of multi-compartment vehicles in which only one type of product (order) is stored in one compartment. Precisely the objective is to propose a genetic algorithm to minimise the total distance and the total cost while making a reasonable choice of vehicles according to the capacity and the number of compartments.

The problem we are presenting is MCV-PDPTW (Multi-Compartment Vehicle for Pickup and Delivery Problem with Time Windows). This MCV-mPDPTW problem is a variant of VRPTW which involves collecting goods from vendors and delivering them to geographically located customers. Also, we have a fleet of $Q_{k}$ capacity $V_{k}$ vehicles which must transport the goods intended for compartments of these vehicles to the customers.

Several works have been carried out on vehicles with several compartments. (El Fallahi et al., 2008) combined a memetic algorithm with a post-optimization phase based on path relinking, and a tabu search method. These algorithms are evaluated by adding compartments to classical VRP instances. 
(Bukchin and Sarin, 2006) have made two approaches, namely dynamic and static, are studied and their performances are compared with each other. In the thesis Optimization of flows: application to distribution problems in animal nutrition, (Cadet, 2013) proposed the algorithms "Greedy Randomized Adaptive Search Procedure" (GRASP) and "Iterated Local Search procedure" (ILS) to solve the MC-VRP with a fixed size compartment dedicated to each product. A new metaheuristic based on a tabu algorithm integrated in a simulated annealing, was developed by (Li et al., 2001) to solve the mPDPTW, this by researching, from the current best solution if there is improvement over multiple iterations. (Curtois et al., 2018) have also proposed a metaheuristic which combines "local search, large neighborhood search and guided ejection search" for the resolution of the PDPTW. (Li et al., 2002) have developed a method called "Squeaky wheel" to solve m-PDPTW with local search .(AlChami et al., 2018) worked on the MuPDPTWPD, a variant of the PDPTW and they combined the Greedy Randomized Adaptive Search Procedure (GRASP) with the Hybrid Genetic Algorithm (HGA) to solve this problem by minimizing the total distance traveled. (Godart, 2019) made a new variant of the PDPTW which authorizes a multiple visit with a storage transfer by proposing a hybrid metaheuristic based on an evolutionary algorithm. (Peng et al., 2018) Worked on the Selective Pickup and Delivery Problem with Time Windows and Paired Demands (SPDPTWPD)". they found the optimal solution using " particle swarm optimization (PSO) and compared it with a 'genetic algorithm. Regarding the m-PDPTW, (Sol et al., 1994) have proposed a branch and price algorithm to solve the m-PDPTW and this by minimizing the number of vehicles necessary to satisfy all the transport demands and the total distance traveled.

This article is divided into four main parts namely: the first part presents the mathematical modeling which aims to serve all customers by minimizing the total cost of transport and respecting time constraints, vehicle capacities and compartments, in the second part we show the complexity of the PDPTW problem, in the third part we will present the MCV and in the fourth part we propose a metaheuristic for the resolution of the MCV-mPDPTW and the results of the simulations as well as the interpretations .

\section{Mathematical model}

In companies, the major challenge is to produce a qualitative service. To do this, the objective is to serve all customer requests, while minimizing the total cost of transport. This cost is relative to the number of vehicles used and the distance traveled by each vehicle.

Our problem is characterized by the following parameters:

- $\quad N$ : Set of customer, supplier and depot nodes,

- $\quad N^{\prime}$ : Set of customer and supplier nodes,

- $\quad N^{+}$: Set of supplier nodes,

- $N^{-}$: Set of client nodes,

- $K$ : Number of vehicles,

- $\quad d_{i j}$ : Euclidean distance between node $i$ and node $j$ if $d_{i j}=\infty$ then the path between $i$ and $j$ does not exist

- $\quad t_{i j k}$ : time taken by the vehicle $k$ to go from node $i$ to node $j$,

- $\left[e_{i} ; l_{i}\right]$ : time window of node $i$,

- $s_{i}$ : time of stop at node $i$

- $\quad q_{i p}$ : quantity of product $p$ to process at node $i$. If $q_{i}>0$, the node is supplier; If

$q_{i}<0$, the node is a client and if $q_{i}=0$ then the node has been served.

- $Q_{k}$ : vehicle $k$ capacity,

- $\quad P$ : all the products delivered to customers

- $W_{p}$ : the capacity of a compartment carrying the product $p$

- $\quad i=0, \ldots, N$ : index of predecessor nodes,

- $j=0, \ldots, N$ : index of successor nodes,

- $k=1, \ldots, N$ : vehicle index,

- $\quad X_{i j k}=\left\{\begin{array}{c}1 \text { if the vehicle } k \text { traveled from the node } i \text { towards the node } j, \\ 0 \text { else }\end{array}\right.$,

- $A_{i}$ : time of arrival at node $i$,

- $D_{i}$ : departure time of the node $i$,

- $y_{i k}$ : quantity present in the vehicle $k$ visiting the node $i$,

- $C_{k}$ : transport cost associated with the vehicle $k$,

- A node (supplier or customer) is only served by one vehicle and at one time, 
- There is only one depot,

- The capacity constraints must be respected,

- The time constraints are rigid regarding the time of arrival,

- Each vehicle begins the journey from the depot and returns there at the end,

- A vehicle remains stationary at a node for the time necessary to process the request.

- If a vehicle arrives at the node $i$ before its window start date $e_{i}$, it waits. (Imen HARBAOUI, 2011),

- The function to be minimized is given as follows:

Minimiser $f=\left(\sum_{k \in K} \sum_{i \in N} \sum_{j \in N} C_{k} d_{i j} X_{i j k}\right)$

$$
\begin{array}{ll}
\sum_{i=1}^{N} \sum_{k=1}^{K} X_{i j k}=1 & j=2, \ldots, N, \\
\sum_{j=1}^{N} \sum_{k=1}^{K} X_{i j k}=1 & i=2, \ldots, N,
\end{array}
$$

$$
\sum_{i \in N} X_{i 0 k}=1 \quad \forall k \in K
$$$$
\sum_{j \in N} X_{0 j k}=1 \quad \forall k \in K,
$$

$$
\sum_{i \in N} X_{i u k}-\sum_{j \in N} X_{u j k}=0 \quad \forall k \in K, \forall u \in N,
$$$$
X_{i j k}=1 \Rightarrow y_{j k}=y_{i k}+q_{i} \quad \forall i, j \in N ; \forall k \in K,
$$$$
y_{0 k}=0
$$$$
\forall k \in K \text {, }
$$

$$
Q_{k} \geq y_{i k} \geq 0
$$$$
\sum_{i \in N} q_{i p}\left(\sum_{i \in N} X_{i u k}\right) \leq W_{p}
$$$$
\forall k \in K, \forall u \in N \text {, }
$$

$D_{w} \leq D_{v}$ $\forall i \in N, \forall w \in N_{i}^{+}, \forall v \in N_{i}^{-}$,

$$
D_{0}=0 \text {, }
$$$$
X_{i j k}=1 \Rightarrow e_{i} \leq A_{i} \leq l_{i}
$$$$
\forall i, j \in N ; \forall k \in K,
$$

$X_{i j k}=1 \Rightarrow e_{i} \leq A_{i}+s_{i} \leq l_{i}$

$\forall i, j \in N ; \forall k \in K$,

$X_{i j k}=1 \Rightarrow D_{i}+t_{i j k} \leq\left(l_{j}-s_{j}\right)$

$\forall i, j \in N ; \forall k \in K$,

Equation (1) represents the objective function that returns the fitness value. It is the minimum total cost related to the total distance travelled by all vehicles in service. Each vehicle has a commissioning cost, which means that the total distance and the total cost are not necessarily proportional.

Equations (2) and (3) ensure that each node is served only once by one and only one vehicle. In this problem we require that a supplier or customer is only visited once by one vehicle. This will allow us to manage our fleet rationally.

Equations (4) and (5) ensure that a vehicle leaves the depot and returns only once. These equations set the number of rounds of a vehicle to 1 and ensure that all vehicles leaving the depot return to the depot only once to avoid under-turning. Subtouring can be considered as a tour that begins at the depot and ends at either a supplier or a customer. These constraints will obviously correct these errors. 
Equation (6) (conservation of flow) ensures the continuity of a tour by a vehicle: the node visited must imperatively be left. This constraint also makes it possible to avoid undertow.

Equations (7), (8) and (9) ensure that the transport capacity of a vehicle is not exceeded. These constraints also ensure that all goods have been delivered. In addition, all vehicles leave the depot with a zero quantity of goods and return to the depot empty. It should also be noted that the quantity of goods collected from each supplier must not exceed the total capacity of the vehicle.

Equation (10) requires that the capacity of the compartment allocated to product $p$ must be at least equal to the sum of the demands for the product. In a compartment we store only one type of product. Each compartment has a capacity. This constraint then requires that the quantity of the product type dedicated to a compartment must be less than or equal to the capacity of that compartment. This will contribute to the choice of vehicles according to the number and capacity of their compartments in the heterogeneous fleet.

Equations (11) and (12) ensure that the precedence is respected. As the suppliers and their respective customers have been identified, our principle is that the goods are collected from the suppliers and delivered to the customers, so an order of passage constraint is imposed. Thus these constraints require that each supplier be visited before his customer by a vehicle.

Equations (13), (14) and (15) ensure that the time windows are respected. Each customer or supplier has the right to define a time interval in which he would like to be visited. Equation (13) requires that the time of arrival of the vehicle at the customer or supplier node must be included in the time window defined by that customer or supplier. This is to avoid long waits or delays at each node. (14) states that the service time on each node must be included in the time window. Finally, (15) notes that the arrival time and service time of the predecessor node must not affect the next node.

\section{Presentation of MCV (Multi-Compartment Vehicle)}

In our problem we will use vehicles with several compartments to produce a quality service. These compartments will be used to store our different products by type. These products can be classified according to the order of loading from suppliers and unloading from customers, while respecting their geographical positions. The quantity of the product type dedicated to a compartment must be less than or equal to the capacity of this compartment. Also, the sum of the quantities of the products must be less than or equal to the total capacity of the vehicle.

Several works have been carried out on the Multi Compartment Vehicle associated with the VRP. These authors have presented these vehicles with their compartments which resolve the problems linked to the transport of incompatible goods. Also they came up with methods for solving their problems. We present some that are: (Ostermeier, 2018) Worked on the MCs associated with the VRP they developed the branch a branch-and-cut (B\&C) algorithm as an exact approach and extend a large neighbourhood search (LNS) as a heuristic approach' for its resolution. (Alinaghian et al., 2018) have proposed a hybrid algorithm composed of adaptive large neighbourhood search and variable neighbourhood search to solve the MCVRP with several repositories in which they seek to minimize the number of vehicles as well as the routes. (Kaabi and Jabeur, 2015) presented multiple compartment vehicles in which they will assign a type of product in a compartment with each client having a time window in which he will be served. they developed a hybrid algorithm including genetic algorithm (GA) and the iterated local search (ILS) is used.
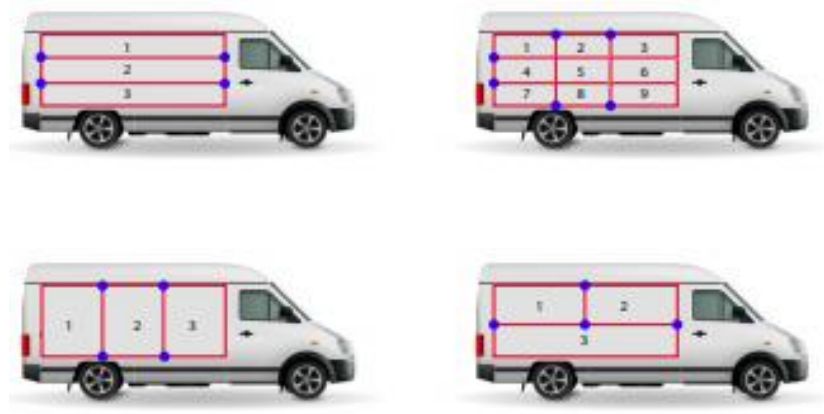

Figure 1. Multi-Compartment Vehicle

\section{Complexity of the PDPTW}

The PDPTW problem we are trying to solve is an NP-Difficult type problem. This PDPTW issue is an extension of VRP that is NP-complete. In the same vein, the VRP is also a classic extension of the Traveling Salesman Problem. This demonstration was made by (LATIF Mehdi, 2018). 


\section{Resolution of the MCV-mPDPTW by the genetic algorithm}

Genetic algorithms were initially developed by (Holland, 1975), (Goldberg, 1989) and (Holland, 1992). They were based on the natural evolution of living things. This algorithm is based on so-called genetic operators to create a population from an initial solution. These are the operators of selection, crossing and mutation of individuals in the population. When we cross two parents, we have the birth of two children. Then we apply the mutation to keep a good level of diversity of the population. Finally, the selection operator is used to eliminate the weakest individuals and the best individual will be the best solution.

The problem that we let's solve is a variant of VRP. This problem consists in minimizing the cost of transport. We consider the MC-PDPTW problem of the following type with:

- A single depot

- A single vehicle has several compartments

- A supplier has several customers

- A customer has several suppliers

- All customers must be served

\subsection{Chromosome coding}

The vehicle has a passage order on each node and must visit all the nodes. This order is stored in a chromosome (Table 1). The vehicle leaves the depot and returns there.

Table 1. Passlist coding

\begin{tabular}{|l|l|l|l|l|l|l|l|l|l|}
\hline Position & Depot & 1ere & 2eme & 3eme & 4eme & 5eme & 6eme & 7eme & Depot \\
\hline Node (i) & D0 & N2 & N4 & N1 & N7 & N6 & N5 & N3 & D0 \\
\hline
\end{tabular}

N1 $=$ Node $(1)$

\subsection{The fitness function}

A function to be optimised: This returns a value called fitness, this is an evaluation function of the individual. In our case, our fitness is the value sent back by $f=\left(\sum_{k \in K} \sum_{i \in N} \sum_{j \in N} C_{k} d_{i j} X_{i j k}\right) \quad$ which is the total cost related to the total distance travelled by the vehicles.

\subsection{Crossovers}

Crossover consists in arbitrarily choosing a crossing point in the chromosomes of the parents. We therefore make combinations of the parents' genes to constitute those of the children, in particular their chromosomes.

Table 2. Crossing in a stitch $\mathrm{p}=3$

Parent 1:

Parent 2:

\begin{tabular}{|l|l|l|l|l|l|l|}
\hline 2 & 6 & 1 & 7 & 4 & 5 & 3 \\
\hline 6 & 1 & 2 & 5 & 3 & 7 & 4 \\
\hline
\end{tabular}

$\mathrm{P}=3$

Child 1:

Child 2:

\begin{tabular}{|l|l|l|l|l|l|l|}
\hline 2 & 6 & 1 & 5 & 3 & 7 & 4 \\
\hline 6 & 1 & 2 & 7 & 4 & 5 & 3 \\
\hline
\end{tabular}

\subsection{Mutation}

The mutation allows to swap two genes to choose randomly in a chromosome by replacing their respective values. The permutation of two genes in a chromosome gives birth to a new individual. The mutation, in our case, particularly allows us to correct the constraints of precedence. When a customer is visited before his supplier, the mutation allows us to make the correction by reversing the order, i.e. to place the supplier before his customer.

Table 3. Sample of mutation operation

\begin{tabular}{l|l|l|l|l|l|l|l|} 
Parent : & G6 \\
Child & G2 & G6 & G1 & G7 & G4 & G5 & G3 \\
\cline { 2 - 8 } & G2 & G4 & G1 & G7 & G6 & G5 & G3 \\
\hline
\end{tabular}

$G=$ gene 


\subsection{Selection}

We make our selection by ranking, which consists of sorting individuals according to their assessment values (fitness). After this ranking, a fixed number of chromosomes is chosen for reproduction.

\subsection{Generation of the initial population}

Our initial population is created to serve as a base for future populations. The choice of this population is very important because it allows for a more or less rapid convergence towards the global optimum.

\subsection{General procedure of genetic algorithm}

Generally, genetic algorithms first generate a population of individuals at random. In order to go from generation $\mathrm{k}$ to generation $\mathrm{k}+1$, the three operations of Crossover, mutation and evaluation are repeated for all the elements of population k. Figure 2 below shows us the general operation of population generation.

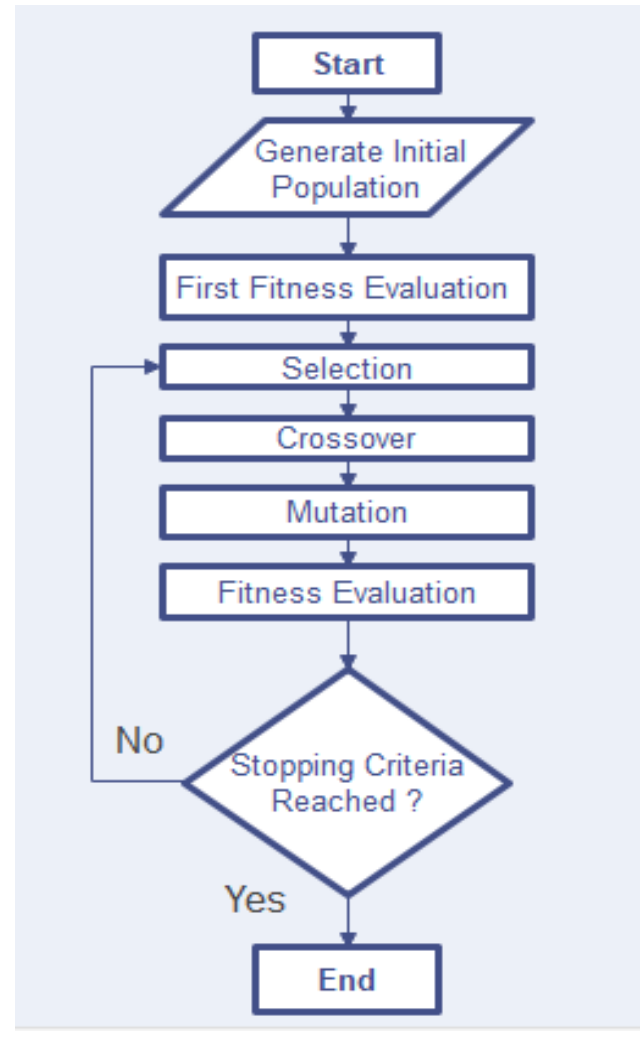

Figure 2. General procedure of genetic algorithm

\subsection{Correction of precedence constraints}

This algorithm makes it possible to correct precedence, that is to say make vehicles visit suppliers before customers (supplier, customer): (F6, C3); (F2, C7) and (F4, C5). We use the mutation so that all suppliers are visited before their respective customers, in case the customer is placed before his supplier the mutation must allow us to make the correction.

Table 4. Sample of correction of precedence constraints

Before correction

Before correction
\begin{tabular}{|l|l|l|l|l|l|l|l|}
\hline $\mathbf{0}$ & F4 & C7 & C3 & F6 & F2 & C5 & 0 \\
\hline
\end{tabular}

After correction
\begin{tabular}{|l|l|l|l|l|l|l|l|}
\hline $\mathbf{0}$ & F4 & F2 & C7 & F6 & C3 & C5 & $\mathbf{0}$ \\
\hline $\begin{array}{l}\mathrm{F}=\text { Supplier } \\
\mathrm{C}=\text { Customer }\end{array}$
\end{tabular}




\subsection{Correction of capacities and compartments constraints}

Either a tour of a vehicle with a total capacity of 90 with three (3) compartments of 30 capacities each. consider the following supplier/customer pairs: F6, C3); (F2, C7); (F4, C5); (F4, C1); (F2, C3); (F6, C7). In this example we show how the constraints of the vehicle compartments work. It is a question of organising the customer orders in the different compartments in such a way as to respect the total capacity of the vehicle and served all the customers.

Customers' orders are listed in the table below:

Table 5. Customer orders from their suppliers

\begin{tabular}{|c|c|c|c|c|c|}
\hline supplier & Product quantities & customer $\mathbf{1}$ & customer $\mathbf{3}$ & customer $\mathbf{5}$ & customer 7 \\
\hline \multirow{2}{*}{ supplier 2} & $q_{1}[2]$ & & & & 30 \\
\hline & $q_{2}[2]$ & & 30 & & \\
\hline \multirow{3}{*}{ supplier 4} & $q_{1}[4]$ & 30 & & & \\
\hline & $q_{2}[4]$ & & & 30 & \\
\hline & $q_{3}[4]$ & & & 30 & \\
\hline supplier 6 & $q_{1}[6]$ & & 15 & & 15 \\
\hline
\end{tabular}

Table 6. Before correction

\begin{tabular}{|l|l|l|l|l|l|l|l|l|}
\hline 0 & F6 & F4 & F2 & C3 & C5 & C7 & C1 & 0 \\
\hline
\end{tabular}

Table 7. Result

\begin{tabular}{|l|l|l|l|l|l|}
\hline & Compartments & F6 & F4 & F2 & Total \\
\hline $\begin{array}{l}\text { vehicle with a total } \\
\text { capacity of 90 with three } \\
\begin{array}{l}\text { (3) compartments of 30 } \\
\text { capacities each }\end{array}\end{array}$ & compartment1 & $\mathbf{1 5 + 1 5 = 3 0}$ & 30 & 30 & 90 \\
\cline { 2 - 6 } & compartment 2 & & 30 & 30 & 60 \\
\cline { 2 - 6 } & compartment3 & & 30 & 30 \\
\hline
\end{tabular}

Table 8. After correction

\begin{tabular}{|l|l|l|l|l|l|l|l|l|}
\hline $\mathbf{0}$ & F6 & F2 & C7 & C3 & F4 & C5 & C1 & 0 \\
\hline
\end{tabular}

Table 9. Result

\begin{tabular}{|l|l|l|l|l|l|l|l|l|l|l|}
\hline & compartments & F6 & F2 & C7 & C3 & F4 & C5 & C1 & Total \\
\hline $\begin{array}{l}\text { vehicle with a total } \\
\text { capacity of 90 with three } \\
\begin{array}{l}\text { (3) compartments of 30 } \\
\text { capacities each }\end{array}\end{array}$ & compartment 1 & $\mathbf{1 5 + 1 5}=\mathbf{3 0}$ & & 15 & 15 & $\mathbf{3 0}$ & & 30 & & \\
\cline { 2 - 10 } & compartment 2 & & $\mathbf{3 0}$ & 30 & & $\mathbf{3 0}$ & 30 & & \\
\cline { 2 - 11 } & compartment 3 & & $\mathbf{3 0}$ & & 30 & $\mathbf{3 0}$ & 30 & & & \\
\hline
\end{tabular}

\subsection{Correction of time constraints}

Time windows are very important in delivery. The example below organizes the time windows in chronological order to respect all the time slots defined by customers and suppliers.

Table 10. Before correction

\begin{tabular}{|l|l|l|l|l|l|l|l|l|}
\hline node (i) & 0 & 5 & 4 & 1 & 7 & 3 & 2 & 6 \\
\hline $\begin{array}{l}\text { time } \\
\text { window } \\
\text { of node } \\
\text { (i) }\end{array}$ & {$[0 ; 24]$} & {$[11 ; 12]$} & {$[17 ; 20]$} & {$[6 ; 7]$} & {$[12 ; 14]$} & {$[7 ; 8]$} & {$[9 ; 11]$} & {$[14 ; 15]$} \\
\hline
\end{tabular}

Table 11. After correction

\begin{tabular}{|l|l|l|l|l|l|l|l|l|l|}
\hline node (i) & 0 & 1 & 3 & 2 & 5 & 7 & 6 & 4 & 0 \\
\hline $\begin{array}{l}\text { Time } \\
\text { window of } \\
\text { node (i) }\end{array}$ & {$[0 ; 24]$} & {$[6 ; 7]$} & {$[7 ; 8]$} & {$[9 ; 11]$} & {$[11 ; 12]$} & {$[12 ; 14]$} & {$[14 ; 15]$} & {$[17 ; 20]$} & {$[0 ; 24]$} \\
\hline
\end{tabular}




\section{Instances and Results}

The problem that we solve is the MCV-mPDPTW (Multi-Compartment Vehicle for Pickup and Delivery Problem with Time Windows). In our program we have a heterogeneous vehicle fleet, each vehicle of which has several compartments. This program is an application of the genetic algorithm in which the vehicles coming from the depot must collect the goods from the suppliers and deliver them to their geographically distributed customers while respecting the time and compartment constraints and must return to the depot.

\subsection{Principle}

In our chromosome we have a deposit, the suppliers and the customers. Our program makes it possible to launch a vehicle which leaves the depot, picks up a supplier, checks if there are other suppliers with whom it has common customers, classifies each order in a compartment of the vehicle and serves the customers before returning at the depot.

\subsection{Results}

Our result table includes four (4) instances 1, 2, 3 and 4 with respectively 25, 50, 75 and 100 nodes. In these instances, we present the solutions with the best fitness. In each instance we present two solutions (routes travelled) according to the minimum total distance travelled, the minimum cost and the number of vehicles used, indicating their characteristics.

Following our simulations, we present our results in the following table:

Table 12. Results of the instances

\begin{tabular}{|c|c|c|c|c|c|c|}
\hline & $\begin{array}{c}\text { Number of } \\
\text { nodes }\end{array}$ & $\begin{array}{c}\text { Number of } \\
\text { vehicles } \\
\text { used }\end{array}$ & $\begin{array}{l}\text { Characteristics of } \\
\text { each vehicle }\end{array}$ & $\begin{array}{c}\text { Minimum } \\
\text { total } \\
\text { distance }\end{array}$ & $\begin{array}{c}\text { Minimum } \\
\text { total cost }\end{array}$ & Paths traveled \\
\hline \multirow[t]{2}{*}{ Instance 1} & 25 & 5 & $\begin{array}{l}\text { V8: } 20 \text { compartments } \\
\text { V9: } 15 \text { compartiments } \\
\text { V11: } 25 \text { compartments } \\
\text { V13: } 15 \text { compartments } \\
\text { V22: } 80 \text { compartments }\end{array}$ & 811147.71 & 6051737.0 & $\begin{array}{c}\text { V8: D1-F36-C42-F44-C41-F49-C47-D1 } \\
\text { V9:D1-F35-C48-F50-C49-C51-C50-F47- } \\
\text { C44-D1 } \\
\text { V11:D1-F51-C52-C53-D1 } \\
\text { V13:D1-F34-C40-C45-D1 } \\
\text { V22: D1-F54-C46-C54-C55-D1 }\end{array}$ \\
\hline & 25 & 4 & $\begin{array}{l}\text { V8: } 20 \text { compartments } \\
\text { V9: } 15 \text { compartiments } \\
\text { V11: } 25 \text { compartments } \\
\text { V13: } 15 \text { compartments }\end{array}$ & 818487.95 & 6608338.0 & $\begin{array}{c}\text { V8: D1-F36-C42-F44-C41-F49-C47-D1 } \\
\text { V9:D1-F35-C48-F47-C44-F50-C49-C51- } \\
\text { C50-D1 } \\
\text { V11:D1-F51-C52-C53-F34-C40-C45-D1 } \\
\text { V22: D1-F54-C46-C54-C55-D1 }\end{array}$ \\
\hline \multirow{2}{*}{ Instance 2} & 50 & 5 & $\begin{array}{l}\text { V8: } 20 \text { compartments } \\
\text { V9: } 15 \text { compartiments } \\
\text { V10: } 18 \text { compartments } \\
\text { V11: } 25 \text { compartments } \\
\text { V22: } 80 \text { compartments }\end{array}$ & 838416.30 & 6896490.9 & $\begin{array}{c}\text { V8 : D1-F36-C42-F44-C41-F49-C47-F58- } \\
\text { C59-F59-C60-F60-C61-F63-C64-D1 } \\
\text { V9: D1-F35-C48-F47-C44-F50-C49-C51- } \\
\text { C50-F56-C57-F57-C58-F62-C63-D1 } \\
\text { V10 : D1-F55-C56-F61-C62-F66-C67-F34- } \\
\text { C40-C45-D1 } \\
\text { V11: D1-F51-C52-C53-D1 } \\
\text { V22 : D1-F67-C68-F54-C46-C54-F64-C65- } \\
\text { F65-C66-D1 }\end{array}$ \\
\hline & 50 & 5 & $\begin{array}{l}\text { V8: } 20 \text { compartments } \\
\text { V9: } 15 \text { compartiments } \\
\text { V10: } 18 \text { compartments } \\
\text { V11: } 25 \text { compartments } \\
\text { V22: } 80 \text { compartments }\end{array}$ & 837606.25 & 6904791.6 & $\begin{array}{c}\text { V8 : D1-F36-C42-F44-C41-F63-C64-F49- } \\
\text { C47-F58-C59-F59-C60-F60-C61-D1 } \\
\text { V9: D1-F35-C48-F50-C49-C51-C50-F56- } \\
\text { C57-F57-C58-F62-C63-F47-C44-D1 } \\
\text { V10 : D1-F34-C40-C45-F55-C56-F61-C62- } \\
\text { F66-C67-D1 } \\
\text { V11: D1-F51-C52-C53-D1 } \\
\text { V22 : D1-F54-C46-C54-F64-C65-F65-C66- } \\
\text { F67-C68-D1 }\end{array}$ \\
\hline Instance 3 & 75 & 5 & $\begin{array}{l}\text { V8: } 20 \text { compartments } \\
\text { V9: } 15 \text { compartiments } \\
\text { V10: } 18 \text { compartments } \\
\text { V11: } 25 \text { compartments } \\
\text { V22: } 80 \text { compartments }\end{array}$ & 849199.42 & $\begin{array}{c}6994526.8 \\
8\end{array}$ & $\begin{array}{c}\text { V8 : D1-F36-C42-F44-C41-F49-C47-F58- } \\
\text { C59-F59-C60-F60-C61-F63-C64-D1 } \\
\text { V9 : D1-F35-C48-F47-C44-F50-C49-C51- } \\
\text { C50-F56-C57-F57-C58-F62-C63-F75-C76- } \\
\text { D1 } \\
\text { V10 : D1-F55-C56-F61-C62-F66-C67-F72- } \\
\text { C73-F73-C74-F79-C80-F34-C40-C45-D1 } \\
\text { V11 : D1-F51-C52-C53-F69-C70-F74-C75- } \\
\text { D1 } \\
\text { V22 : D1-F80-C81-F54-C46-C54-F64-C65- } \\
\text { F65-C66-F67-C68-F68-C69-F70-C71-F71- } \\
\text { C72-F76-C77-C78-F78-C79-D1 }\end{array}$ \\
\hline
\end{tabular}




\begin{tabular}{|c|c|c|c|c|c|c|}
\hline & $\begin{array}{c}\text { Number of } \\
\text { nodes }\end{array}$ & $\begin{array}{l}\text { Number of } \\
\text { vehicles } \\
\text { used }\end{array}$ & $\begin{array}{l}\text { Characteristics of } \\
\text { each vehicle }\end{array}$ & $\begin{array}{c}\text { Minimum } \\
\text { total } \\
\text { distance }\end{array}$ & $\begin{array}{c}\text { Minimum } \\
\text { total cost }\end{array}$ & Paths traveled \\
\hline & 75 & 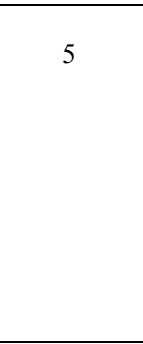 & $\begin{array}{l}\text { V8: } 20 \text { compartments } \\
\text { V9: } 15 \text { compartiments } \\
\text { V10: } 18 \text { compartments } \\
\text { V11: } 25 \text { compartments } \\
\text { V22: } 80 \text { compartments }\end{array}$ & 854357.23 & 7058725.9 & $\begin{array}{c}\text { V8 : D1-F36-C42-F44-C41-F49-C47-F58- } \\
\text { C59-F59-C60-F60-C61-F63-C64-D1 } \\
\text { V9 : D1-F35-C48-F50-C49-C51-C50-F56- } \\
\text { C57-F57-C58-F62-C63-F75-C76-F47-C44- } \\
\text { D1 } \\
\text { V10 : D1-F34-C40-C45-F79-C80-F55-C56- } \\
\text { F61-C62-F66-C67-F72-C73-F73-C74-D1 } \\
\text { V11 : D1-F51-C52-C53-F69-C70-F74-C75- } \\
\text { D1 } \\
\text { V22 : D1-F54-C46-C54-F64-C65-F65-C66- } \\
\text { F67-C68-F68-C69-F70-C71-F71-C72-F76- } \\
\text { C77-C78-F78-C79-F80-C81-D1 } \\
\end{array}$ \\
\hline \multirow[b]{2}{*}{ Instance 4} & 100 & 7 & $\begin{array}{l}\text { V8: } 20 \text { compartments } \\
\text { V9: } 15 \text { compartments } \\
\text { V10: } 18 \text { compartments } \\
\text { V11: } 25 \text { comparments } \\
\text { V2: } 55 \text { compartiments } \\
\text { V22: } 80 \text { compartments } \\
\text { V25: } 3 \text { compartments }\end{array}$ & 851921.15 & 8406999.6 & $\begin{array}{c}\text { V8 : D1-F49-C47-F58-C59-F59-C60-F60- } \\
\text { C61-F63-C64-D1 } \\
\text { V9 : D1-F47-C44-F57-C58-F62-C63-F75- } \\
\text { C76-F100-C107-D1 } \\
\text { V10 :D1-F35-C48-C102-F56-C57-C95- } \\
\text { C101-F61-C62-F66-C67-F72-C73-F73- } \\
\text { C74-F79-C80-F81-C82-F92-C92-F34-C40- } \\
\text { C45-D1 } \\
\text { V11 : D1-F36-C42-C96-F50-C49-C51-C50- } \\
\text { C98-F51-C52-C53-F69-C70-F74-C75-D1 } \\
\text { V21 : D1-F44-C41-C97-D1 } \\
\text { V22 : D1-F55-C56-C100-F64-C65-F65- } \\
\text { C66-F67-C68-F68-C69-F70-C71-F71-C72- } \\
\text { F76-C77-C78-F78-C79-F80-C81-F95- } \\
\text { C103-F97-C104-D1 } \\
\text { V25 : D1-F93-C93-F91-C91-F98-C105- } \\
\text { C106-D1 }\end{array}$ \\
\hline & 100 & 8 & $\begin{array}{l}\text { V8: } 20 \text { compartments } \\
\text { V9: } 15 \text { compartments } \\
\text { V10: } 18 \text { compartments } \\
\text { V11: } 25 \text { compartments } \\
\text { V21: } 55 \text { compartments } \\
\text { V22: } 80 \text { compartments } \\
\text { V24: } 35 \text { compartments } \\
\text { V25: } 3 \text { compartments }\end{array}$ & 855167.26 & 8490166.7 & $\begin{array}{c}\text { V8 : D1-F49-C47-F58-C59-F59-C60-F60- } \\
\text { C61-F63-C64-D1 } \\
\text { V9 : D1-F47-C44-F57-C58-F62-C63-F75- } \\
\text { C76-F100-C107-D1 } \\
\text { V10 : D1-F35-C48-C102-F56-C57-C95- } \\
\text { C101-F61-C62-F66-C67-F72-C73-F73- } \\
\text { C74-F79-C80-F81-C82-F92-C92-F34-C40- } \\
\text { C45-D1 } \\
\text { V11 : D1-F36-C42-C96-F50-C49-C51-C50- } \\
\text { C98-F51-C52-C53-F69-C70-F74-C75-D1 } \\
\text { V21 : D1-F44-C41-C97-D1 } \\
\text { V22 : D1-F97-C104-F55-C56-C100-F64- } \\
\text { C65-F65-C66-F67-C68-F68-C69-F70-C71- } \\
\text { F71-C72-F76-C77-C78-F78-C79-F80-C81- } \\
\text { F95-C103-D1 } \\
\text { V24 : D1-F54-C46-C54-C99-D1 } \\
\text { V25 : D1-F91-C91-F93-C93-F98-C105- } \\
\text { C106-D1 }\end{array}$ \\
\hline
\end{tabular}

\subsection{Interpretation of the results}

- In the first instance we present two solutions. The first uses 5 vehicles with a minimum total distance and a minimum total cost while the second uses 4 vehicles with a higher distance and cost, this is explained by the cost of commissioning the vehicles, the distances travelled by each vehicle and by compartment constraints.

- In the second instance, the costs of the vehicles and the distances travelled by each vehicle give us a large distance with a smaller cost in the first solution while in the second solution we have a small distance with a high cost.

- In the third instance, we have a minimum distance with a minimum cost and a large distance with a high cost in the first and second solution. This shows that the total cost and the total distance travelled by all vehicles are not proportional.

- In the last instance, unlike the first, we have a reduced number of vehicles used showing a minimum cost in the first solution and in the second solution a high number of vehicles used showing a slightly higher cost. It depends on the costs of commissioning and their chosen routes. In these instances, the vehicles used are not the same because the choices are made according to the products (orders) available and the compartments.

\section{Conclusion}

In this article we have presented the MCV-mPDPTW problem that we want to solve. Also, we established the mathematical model and showed the complexity of the problem. We have developed a 
program based on the genetic algorithm which solves our problem giving the optimal solution and minimal cost with a very reduced computation time. The more we have a large number of nodes also the computation time becomes great. We can apply our results in companies specialized in the delivery of goods.

\section{Acknowledgment}

We would like to thank ESATIC for facilitating our research and allowing access to data. Our thanks also go to his dynamic team and to all the other persons in charge of the said structure. We remain grateful to the research laboratory that welcomes us within the African School of ICT: LASTIC.

\section{References}

1. El Fallahi, A., Prins, C. et Wolfler Calvo, R. (2008) A memetic algorithm and a tabu search for the multi-compartment vehicle routing problem, Computers \& Operations Research, 35(5):1725-1741.

2. AlChami, et al. (2018) An advanced GRASP-HGA combination to solve a multi-period Pickup and Delivery Problem, Expert Systems with Applications, 105, March 2018.

3. Godart, A. (2019) Hybrid metaheuristic for the Pickup and Delivery Problem designed for passengers and goods transportation, IFAC-PapersOnLine, 52(13): 2584-2589, January 2019.

4. Joseph, Cadet David. (2013) Optimisation des flux: application aux problèmes de distribution en nutrition animale, 2013TROY003, 18 décembre 2013.

5. Goldberg, D. E. (1989) Genetic Algorithms in Search, Optimization, and Machine Learning. Addison-Wesley Professional, 1 edition, 1989. ISBN 0201157675.

6. Kaabi, Hadhrami and Jabeur, Khaled (2015) International Conference on Informatics in Control, Automation and Robotics (ICINCO), Institute of Electrical and Electronics Engineers, pp. 21-23, July 2015.

7. Li, H., Lim, A. (2001) A metaheuristic for the pickup and delivery problem with time windows. In: IEEE International Conference on Tools with Artificial Intelligence, 13, 160-167.

8. Li, H., Lim, A., Rodrigues, B. (2002) Solving the pickup and delivery problem with time windows using squeaky wheel" optimization with local search. SMU Business Conference Paper Series.

9. Alinaghian, Mahdi, et al. (2018) A Hybrid Algorithm Composed Of Adaptive Large Neighbourhood Search and Variable Neighbourhood Search PDPTW, Elsevier, 76, 85-99, April 2018.

10. Harbaoui Dridi, Imen. (2011) Optimisation heuristique pour la résolution du m-PDPTW statique et dynamique, Int. J. of Computers, Communications \& Control, ISSN 1841-9836, E-ISSN 1841-9844, Vol. VI, 246-257.

11. Holland, J. H. (1975) Adaptation in natural and artificial systems. The University of Michigan Press, Ann Arbor.

12. Holland, J. H. (1992) Adaptation in Natural and Artificial Systems: An Introductory Analysis with Applications to Biology, Control, and Artificial Intelligence. Bradford Books. MIT Press. ISBN 9780262581110.

13. Latif, Mehdi. (2018) Conception d'une métaheuristique pour la résolution du Pickup and Delivery Problem with Time Windows, pp. 7-8, 4 juin 2018.

14. Ostermeier, M. (2018) Loading constraints for a multi-compartment vehicle routing problem, $O R$ Spectrum, 40, 997-1027.

15. Sol, M., Savelsbergh, M. (1994) A branch-and-price algorithm for the pickup and delivery problem with time windows. Memorandum COSOR 94-22, Dept. of Mathematics and Computing Science, Eindoven University of Technology, Eindoven, Netherlands.

16. Kammarti, R. (2007) Approches Evolutionnistes Pour La Resolution Du 1-Pdptw Statique Et Dynamique, https://tel.archives-ouvertes.fr/tel-00143782, 25 mai 2007.

17. Curtois, T. et al. (2018) Large neighborhood search with adaptive guided ejection search for the pickup and delivery problem with time windows, EURO Journal on Transportation and Logistics, 7 , 151-192.

18. Bukchin, Y., Sarin, Subhash C. (2006) Discrete and dynamic versus continuous and static loading policy for a multi-compartment vehicle, European Journal of Operational Research, 174(2), 1329-1337.

19. Peng, Zh. et al. (2018) A Particle Swarm Optimization for Selective Pickup and Delivery Problem, Conference Paper, November 2018. 